\title{
La Toma de Decisiones Eticas en Ginecología y Obstetricia.
}

Traducción del Boletín Técnico. "Una ayuda educativa para los Obstetras-Ginecólogos”. Número 136, noviembre 1989. Colegio Americano de Obstetras y Ginecólogos (ACOG)

Los progresos recientes en la tecnología científica han aumentado las posibilidades disponibles para el tratamiento de varias condiciones médicas. También ha aumentado la capacidad de la medicina para intervenir en los procesos del nacimiento, la vida y la muerte.

Lo anterior es más aparente en obstetricia y ginecología que en cualquiera otro campo.

Las capacidades técnicas para la "reproducción ayudada" la cirugía fetal, y las intervenciones al final de la vida, suscitan interrogantes que no pueden ser contestados únicamente con base en el conocimiento médico. Las decisiones correctas en este campo exigen la consideración cuidadosa de los valores, los deseos y las metas de quienes están comprometidos en dicha decisión. Los médicos, como resultado de su educación médica formal son muy diestros en la toma de decisiones basadas en indicaciones médicas. Es muy importante también que ellos adquieran destrezas para enfrentar decisiones cuando se enfrentan valores en conflicto o metas personales diferentes.

La Comisión Presidencial para el Estudio de los Problemas Eticos en Medicina ha afirmado que "la primera responsabilidad para garantizar que se tomen decisiones moralmente justificadas reposa en el médico"(1). Sin embargo, antes de llevar a cabo una aproximación ética para enfrentar problemas difíciles, es indispensable tener en cuenta ciertos aspectos fundamentales (2):

1. El médico debe tener una idea muy clara de la estructura de su propio sistema de valor y de la forma en que sus juicios personales acerca de lo que es bueno o malo, influyan las decisiones en varios aspectos de la vida.
2. El médico debe tener un conocimiento básico de la ética como disciplina.

3. El proceso por el cual el médico llega a las decisiones éticas y las implementa, debe ser sistemático, consistente con la lógica.

\section{VALORES, MORAL Y ETICA}

Cada individuo, como resultado de una variedad de experiencias vitales, adquiere un sentido personal de lo que tiene valor.

Ciertos objetos, relaciones y resultados son más deseables que otros. Estos valores sirven a manera de prisma a través del cual la información se refleja antes de ser interpretada y aplicada a las decisiones de la vida. Los valores sirven también de cimiento a las reglas por medio de las cuales los individuos manejan sus propias acciones.

Lo moral se puede describir como el pensamiento común de lo que es bueno o malo, de lo que debe o no debe ser hecho. Al enfrentar las decisiones simples de cada día, este pensamiento del bien y el mal, idealmente fundamentado en un sólido sistema de valores y principios, le será usualmente muy útil al individuo que busque soluciones razonables. Cuando los interrogantes comienzan a requerir juicios basados en los valores de varios grupos con diversos puntos de vista y metas contrarias, las determinaciones personales acerca de lo que es bueno o malo son inadecuadas para alcanzar una solución. Se hace necesaria una aproximación lógica y disciplinada (sistemática) para lograr una decisión válida a problemas complejos. La ética puede proporcionar esa aproximación.

La ética es el estudio formal del comportamiento moral en el cual las obligaciones morales son analizadas en términos de principios éticos reconocidos. Después de un cuidadoso análisis se hace un intento para determinar cual, entre varias supuestas morales es justifica- 
ble. La ética es una disciplina que ha evolucionado a partir de este análisis crítico y de la discusión de asuntos morales en el curso de muchos siglos de la civilización. Figuras históricas prominentes en filosofía moral como Platón, Aristóteles, Tomás de Aquino, Imanuel Kant y John Stuart Mill han hecho importantes contribuciones a esta disciplina.

Un dilema ético puede surgir cuando justificaciones basadas en ciertos valores parecen apoyar dos o más maneras de actuar que son contradictorias. Los principios fundamentales de como deben pensar y actuar los individuos, pueden parecer apoyando enfoques encontrados al problema en cuestión. Inicialmente los dos argumentos o líneas de acción pueden aparecer igualmente atractivos. Por ejemplo, el médico que enfrenta a una mujer que rechaza tratamiento quirúrgico de una gran masa pélvica se sentirá ambivalente entre su obligación de aconsejar lo que parece ser más adecuado para los intereses médicos de la paciente por una parte y su deber de respetar la decisión de ella para rechazar el tratamiento.

Los conflictos éticos también pueden surgir de la falta de acuerdo entre quienes deben tomar una decisión, acerca de los valores en que deben basarse sus juicios. Una fuente muy importante de dichos conflictos son las creencias personales o las políticas que el médico o la institución introduce en el proceso de decisión. Estas creencias personales y políticas institucionales deben ser entendidas y reconocidas antes de iniciar un análisis objetivo del problema.

Por ejemplo un médico puede apoyar a una mujer en su deseo de obtener tratamiento para su infertilidad mientras que otro, el administrador de la institución de salud, puede estar mucho más interesado en proporcionar servicios de salud a bajo costo para un gran número de personas.

$\mathrm{Al}$ aplicar reglas y principios morales a las acciones humanas, la ética no identifica ningún principio moral como el único correcto. En lugar de esto, sirve como marco de referencia para el análisis sistemático de diferentes puntos de vista morales. Al desarrollar este análisis, uno pretende lograr una justificación racional para una manera de actuar en lugar de otra sobre la base de valores y principios comúnmente aceptados.

\section{PRINCIPIOS ETICOS}

$\mathrm{Al}$ hacer el análisis ético de un problema, se invocan con frecuencia principios como justificación de las reglas que dirigen las acciones. Obligaciones que pueden ser antagónicas en relación con la salud, se pueden aclarar cuando se entienden y se identifican los princi- pios subyacentes. Los principios comúnmente aplicados en discusiones acerca de la ética son los de autonomía, caridad (o humanitarismo) y justicia.

\section{AUTONOMIA}

La autonomía se define como una regla automática. La persona es libre de establecer normas de conducta personales y de escoger voluntariamente el camino a seguir con base en una serie de valores personales consistentes y de principios emanados de éstos. Esta libertad impone en los demás una obligación de no-interferencia. La autonomía es considerada por algunos como algo casi absoluto a menos que viole la libertad personal de otros. Otros puntos de vista imponen límites más estrechos en la autonomía individual.

El respeto por la autonomía juega un papel importante en la práctica médica. Si bien el médico puede considerar un tipo de tratamiento como el mejor para su paciente, también puede estar de acuerdo con un manejo un poco diferente de acuerdo con la solicitud del paciente. Como otro ejemplo, el médico que aconseja el evitar un futuro embarazo a una paciente con historia clínica de cardiomiopatía postpartum eventualmente mortal, por respeto a la autonomía de ésta, se abstiene de tomar acciones que interfieran con su opción reproductiva.

\section{CARIDAD (HUMANITARISMO)}

Humanitarismo es la obligación de promover el bienestar de los demás. El principio relacionado de no hacer el mal obliga a la persona a evitar, herir o perjudicar a otros. Con raíces en la tradición Hipocrática, el hacer el bien y evitar el mal, son fundamentales a la ética médica. La aplicación de estos principios consisten en equilibrar los beneficios y los daños. En este contexto, el significado de "daño" incluye los daños o perjuicios intencionales así como aquellos que se pueden prever o que pueden surgir a pesar de las mejores intenciones (por ejemplo, efectos secundarios iatrogénicos o complicaciones de un tratamiento quirúrgico). Por lo tanto estos principios son la fuente de la obligación médica de actuar con debidas precauciones.

El humanitarismo (o bondad) fundamenta la obligación del médico de promover el bienestar de una mujer con salpingitis mediante la administración de antibióticos por vía intravenosa. Equilibrando el humanismo con la autonomía uno busca identificar "lo que es mejor" para su paciente tan objetivamente como es posible. Los esfuerzos para supeditar la autonomía del paciente a lo que el médico considera como lo más indicado desde su punto de vista se llama paternalismo. 


\section{JUSTICIA}

La justicia es el derecho de los individuos para reclamar lo que les pertenece (o tienen derecho) basándose en ciertas propiedades o características personales. Algunas teorías del principio de Justicia resuelven la distribución de los beneficios y los perjuicios (o daños) basándose en propiedades específicas, utilizando criterios como la necesidad, el esfuerzo, la contribución (colaboración) o el mérito. Otras teorías especifican que todos los "costos" y los beneficios se deben distribuir equitativamente (o por igual). Es importante que el criterio utilizado haya sido de una manera consistente con reglas y principios morales aceptados y que estos estén directamente relacionados con los "costos" y los beneficios que se están distribuyendo o asignando. En nuestra sociedad, por ejemplo, raza, sexo y religión, no se consideran criterios morales legítimos para la distribución de beneficios tales como el empleo y la habitación. El principio de justicia genera la obligación de tratar lo mismo a aquellos que son iguales de acuerdo con cualquier criterio que se haya utilizado. Ninguna persona puede recibir tratamiento desigual a menos que se demuestre que él o ella son diferentes a otros, en una forma que se relacione con la conducta en consideración.

A veces, límites tales como la escasez de recursos imponen juicios morales acerca de reclamaciones en conflicto hechas por quienes parecen estar basados en criterios previamente utilizados. En este caso se deben seleccionar criterios diferentes a partir de los cuales se pueda llevar a cabo la distribución de los beneficios. La selección de estos criterios representa en sí misma una decisión moral. Una situación común se presenta con un paciente en estado crítico y la unidad de cuidados intensivos (UCI) que está totalmente copada. Todos los pacientes en la UCI fueron referidos a esta por que se consideraba que su estado lo requería. Pero cuando se presenta una escasez de camas, es necesario utilizar otros criterios para decidir que pacientes requieren dichas camas "con mayor urgencia". La discusión de tales situaciones se centra con frecuencia en las probabilidades de recuperación exitosa y en los riesgos para el paciente que sea trasladado a un medio menos especializado.

\section{CONCEPTOS ETICOS}

Varias normas éticas pueden derivarse de estos principios éticos fundamentales. Estas normas o conceptos son importantes por que ellos se aplican a muchas de las decisiones que hacemos en obstetricia y ginecología. La comprensión de estos conceptos aumentan la probabilidad de que las decisiones sean consistentes con los principios éticos aceptados.

\section{Consentimiento informado}

El consentimiento informado puede definirse como "la aceptación voluntaria, no forzada, de una intervención médica por el paciente luego de adecuada explicación por el médico de la naturaleza de la intervención, sus riesgos y beneficios" (4). El propósito primordial del proceso de consentimiento es la protección de la autonomía del paciente. Al estimular una comunicación permanente y abierta, el médico le permite al paciente llevar a cabo su decisión personal. Este tipo de comunicación es fundamental en la relación médico-paciente.

Es importante reconocer que el derecho del paciente a tomar sus propias decisiones en asuntos de salud se extiende a su derecho a rechazar el tratamiento médico ordenado. Esta libertad de aceptar o rechazar un tratamiento esta apoyada legal y éticamente. Por ejemplo, el Juez Benjamín Cardozo declaró en 1914 que, "todo ser humano adulto y en sus cabales tiene el derecho a decidir lo que se haga con su propio cuerpo" (5).

A veces la capacidad del paciente para entender y procesar la información médica presentada a ella puede estar en duda. El médico, consultando y discutiendo con la paciente, debe intentar aclarar y determinar cual es su capacidad para dar el consentimiento solicitado. Si un paciente es incapaz de dar consentimiento, se debe buscar quién pueda hacerlo a su nombre.

\section{Honestidad}

El principio de autonomía requiere que se dé al paciente una información verdadera y completa acerca de su condición médica y acerca de cualquier tratamiento propuesto. Unicamente con esta información será ella capaz de ejercitar su derecho a tomar decisiones acerca de su salud. Si la información completa no está disponible para el médico, las dudas existentes deben ser compartidas con la paciente. Es inadecuado que el médico asuma estar mejor capacitado para determinar lo que la paciente quisiera saber, que la paciente misma. En general la paciente se beneficia de conocer en detalle su situación de salud, su pronóstico y los tratamientos disponibles. La sospecha de que el médico ha ocultado la verdad o lo ha engañado, debilitará la fe del paciente y socavará la relación médico-paciente. Por lo tanto la norma de honestidad puede estar fundamentada en el principio de beneficencia así como en el principio de autonomía.

\section{Confidencialidad}

El derecho del paciente a tomar decisiones en relación con su salud incluye el derecho a decidir cómo y a quién se le debe comunicar la información médica relativa a él. El principio de autonomía fundamenta el deber del médico de respetar la privacidad del paciente. 
Además el paciente de la protección de su derecho a la confidencialidad. Igual que con la deshonestidad, fallas en la confidencialidad amenazan la confianza del paciente en su médico y pueden destruir la relación médico-paciente.

Pueden surgir situaciones en las cuales el mantener la confidencialidad del paciente puede resultar en perjuicio para terceras personas. Por ejemplo una paciente puede negarse a que se informe a su marido de una enfermedad de trasmisión sexual que ella adquirió durante una relación extramarital. El médico enfrenta entonces un dilema ético: La obligación de respetar la autonomía de la paciente entra en conflicto con la obligación de evitar daño a otros que están en riesgo. Un examen de los principios encontrados y de las reclamaciones que pueden surgir de ellos ayudará al médico a decidir sobre que camino debe seguir. En general, la obligación de mantener la confidencialidad tiene prioridad sobre otras obligaciones.

\section{RELACIONES EN LA TOMA DE DECISIONES}

La naturaleza de las relaciones entre las diferentes partes interesadas en decisiones sobre la salud tiene un impacto importante en las decisiones mismas. Las metas y los valores de las personas pueden no estar de acuerdo. Una decisión que puede beneficiar a una de las partes puede resultar en un perjuicio relativo para otra. La comprensión de las relaciones entre las partes involucradas en un dilema ético es esencial cuando se pretende llegar a una solución ponderada.

\section{Relación Médico-Paciente}

La forma en que se pesan los principios al examinar asuntos éticos dependerá de como están definidas las relaciones médico-paciente y las metas médicas. Lo fundamental para esta definición es la decisión de si el mayor beneficio para el paciente se une desde el punto de vista médico o desde el punto de vista del paciente. El sistema de valores de quienes se enfrentan a una decisión ética influenciará la forma en la cual se pesan principios y normas en contraposición y puede por lo tanto ser fuente de conflicto.

En general los médicos consideran el principio de beneficencia (de hacer siempre el bien) como su obligación más importante. Partiendo de una orientación médica ellos dan especial valor al bienestar físico y escogen por lo tanto las acciones que puedan promoverlo. Las molestias personales o económicas pueden ser vistas por este médico como "costos" razonables en el camino hacia una mejor salud. El modelo dominado por el principio de beneficencia en las relaciones médico-paciente busca lo que es mejor para este último desde el punto de vista médico. De acuerdo con este modelo las metas y los valores de la medicina tiene una importancia primordial en el momento de decidir lo que es mejor para el paciente. El médico que actúa de acuerdo con este modelo puede hacerlo en forma paternalista sin tener en cuenta las preferencias del paciente con el objeto de promover lo que él cree que más conviene al paciente.

En contraste el modelo basado en el principio de autonomía, que busca promover los intereses del paciente de acuerdo con su punto de vista, más probablemente asegurará que los valores y creencias del paciente queden salvaguardados en las decisiones que se tomen. Puede que el paciente no vea que es "bueno" en la misma forma que el médico. La curación puede que no sea su meta más importante, o los costos de alcanzar la salud pueden parecerle demasiado altos. Al tomar decisiones acerca de tratamientos médicos algunas personas darán importancia primordial a consideraciones familiares o religiosas antes que a la búsqueda de su salud física. El conflicto ético puede disminuirse si el médico es capaz de fijar las metas del tratamiento de acuerdo con los valores del paciente. En raras ocasiones el médico puede sentirse impedido moralmente para cooperar en la búsqueda de objetivos médicos de un paciente en particular. En este caso deberá recomendar alternativas apropiadas.

\section{Relaciones Materno Fetales}

En el cuidado de la paciente obstétrica, la relación tradicional médico-paciente se extiende al feto. El médico conserva la obligación de respetar la autonomía de la mujer embarazada y al mismo tiempo busca su bienestar médico (o de salud?). Además el médico debe aceptar su obligación de beneficencia (de hacer el bien) a un segundo paciente, el feto. El peso que se da a este deber en comparación con deberes previamente establecidos para con la mujer es crucial a muchos dilemas médicos en la práctiça de la obstetricia.

El médico puede interpretar lo que es mejor para el feto, sea de acuerdo con el punto de vista y los valores de la mujer embarazada, en un esfuerzo por respetar su autonomía, o de acuerdo con sus propios valores y punto de vista como médico. Ambas perspectivas o puntos de vista pueden hacer contribuciones importantes al proceso de toma de decisiones. Frecuentemente la mujer y el médico están de acuerdo con lo que es mejor para la mujer y para el feto. Sin embargo, a veces las conclusiones de la mujer sobre los riesgos y beneficios relativos se apartan diametralmente de los del médico. En razón del respeto a su autonomía, se considera a la mujer como la fuente del consentimiento del feto que ella lleva consigo. El concepto de la mujer, sobre lo que es mejor (el beneficio, para el feto determina generalmente el tratamiento a realizar. En la ma- 
yoría de las situaciones, las mujeres embarazadas están dispuestas a grandes esfuerzos para promover el bienestar de sus futuros hijos.

Muy rara vez, una situación particularmente difícil puede surgir cuando una mujer embarazada descuida su obligación de proteger su feto al rechazar un tratamiento que puede salvar su vida o al asumir comportamientos peligrosos para la salud del feto. El obstetra debe hacer un esfuerzo a través de discusión y consulta para que la mujer tome conciencia de las implicaciones que sus actos tienen sobre la salud del feto. Aunque se puede estar de acuerdo con que la mujer embarazada tiene obligación fundamental sobre el feto, ninguna otra parte interesada incluyendo el estado, debe supeditar su autonomía con el objeto de imponer esas obligaciones o deberes (6).

\section{Relaciones con Terceras Personas o Entidades}

$\mathrm{Si}$ bien los intereses de la paciente y su médico usualmente determinan las decisiones médicas, los intereses de "terceras partes" deben tenerse en cuenta a veces. Las peticiones de estas terceras partes estan basadas frecuentemente en principios o normas aceptados. A pesar de que la mayoría de los médicos se sienten muy inclinados a respetar la confidencialidad de sus pacientes, pueden también pensar hasta donde su deber de beneficencia se extiende a terceras personas. Este dilema puede surgir cuando el conocimiento que el médico tiene de su paciente incluye información acerca de enfermedades genéticas o infecciosas que representen un riesgo para otros.

En la obstetricia el padre puede exigir que la mujer se someta a un tratamiento en beneficio del feto. Esta exigencia puede estar basada en su propia autonomía así como en su deseo de beneficiar al feto (y por lo tanto a su futuro hijo-a). Aunque las decisiones obstétricas son todavía la prerrogativa de la mujer embarazada y de su médico, éste puede enfrentarse a una tercera persona que exige tener también un derecho.

Las metas de los servicios de salud difieren a veces de aquellas de los pacientes o médicos individuales. Asuntos como la capacidad financiera pueden entrar en conflicto con la necesidad de hacer una distribución adecuada de los servicios. Si bien los individuos que trabajan en las instituciones de salud o los empleados por otros proveedores de servicios pueden hasta cierto punto adoptar los valores de dichas instituciones, pueden presentarse conflictos entre los propios valores del individuo o de su profesión y aquellos de la institución para la cual trabaja. Por ejemplo pueden existir presiones para reducir costos mediante la restricción de algunos procedimientos (diagnósticos o terapéuticos) con miras a que todos los pacientes puedan tener acceso a servicios básicos de salud. Si bien el médico puede entender la razón de ser de las políticas institucionales y de sus decisiones, él o ella se sentirán frustrados por los esfuerzos inútiles de lograr un cierto nivel de servicios de salud. La potencialidad de conflicto puede disminuirse, si los valores institucionales son claramente comunicados a aquellos de quien se espera los respeten.

En la mayoría de las situaciones, las decisiones médicas serán tomadas por el paciente individual con el consejo y recomendación de su médico. No obstante, a veces, personas o instituciones que estrictamente no forman parte del binomio médico-paciente harán exigencias que serán razonables. El que dichas exigencias sean decisorias cuando se enfrenta un serio problema, dependerá de un examen minucioso de los principios éticos involucrados en cada caso.

\section{GUIAS PARA LA TOMA DE DECISIONES}

Con frecuencia, más de un solo camino a seguir puede ser moralmente justificable a partir del examen de principios morales. A veces parecerá que ningún camino a seguir es aceptable puesto que cada uno puede ocasionar perjuicios considerables. No obstante, una de las posibles opciones tiene que ser escogida y su selección debe estar apoyada en un razonamiento ético. Los intentos para resolver estas dificultades pueden ser ayudados por un análisis racional de los varios factores involucrados. Por otra parte, la participación de individuos con antecedentes y puntos de vista diferentes pueden ser útiles cuando se consideran asuntos de ética. La consulta con personas de servicios relacionados o semejantes, o con comités hospitalarios de ética pueden ser de gran ayuda en garantizar que todos los puntos de vista han sido analizados cuando se toma una decisión (7).

Es importante para cada médico desarrollar un esquema para su toma de decisiones que pueda aplicar siempre cuando se enfrente a dilemas éticos. Una aproximación puede consistir en una serie de pasos lógicos que le puedan ayudar a manejar el problema ético. Se han propuesto varios esquemas muy útiles $(2,4,8,9)$ de los cuales se ha extraido los elementos aquí presentados.

1. Identifique quienes son los que están tomando la decisión.

El primer paso es contestar la pregunta "¿Quién debe tomar la decisión?". En general, el poder decisorio final reposa en el paciente.

a. A veces la capacidad del paciente para tomar decisiones no es muy clara. La capacidad de una persona para tomar decisiones depende 
de su habilidad para entender y usar la información, basándose en un sistema coherente de valores para poder llegar a una decisión sobre el camino a seguir. En contraste, la capacidad o incapacidad son resoluciones legales que puieden o no reflejar fielmente la capacidad funcional. La evaluación de la capacidad del paciente para tomar decisiones tiene en ocasiones que ser hecha por profesionales expertos en dichas determinaciones. Las decisiones acerca de la capacidad pueden ser tomadas únicamente en una Corte de Justicia.

b. Si se piensa que el paciente es incapaz de tomar una decisión o legalmente se ha determinado así, habrá que identificar un substituto. Esta persona debe estar en capacidad de actuar y promover lo que mejor convenga al paciente. El médico no se convierte automáticamente en dicho poder decisorio. Tradicionalmente se ha llamado a los familiares a ejercer dichas funciones. En algunas situaciones, se puede acudir a la corte para que designe un guardián o protector. Sin embargo, el médico tiene la obligación de ayudar a quienes representan al paciente a examinar el asunto y llegar a una decisión.

c. Existen dos criterios primordiales de acuerdo con los cuales se toman las decisiones por delegación. En los casos donde existe información que sugiere lo que el paciente hubiera escogido si estuviera en capacidad de hacerlo, se podrá ofrecer un juicio substituto. Este proceso es lo que más se acerca a respetar la autonomía de la persona involucrada. En algunas partes, este concepto ha sido formalizado mediante un testamento o mediante un poder especial a un abogado. Si el juicio por delegación no es posible por que no existen afirmaciones previas por parte del paciente, el substituto debe tomar la decisión que promueva los mejores intereses del paciente. Cualquier información existente acerca del paciente ayudará a definir lo que esa persona hubiera considerado como lo mejor.

d. En el campo obstétrico, se considera a la mujer embarazada como decisor adecuado para el feto que lleva consigo.

2. Obtenga datos, establezca los hechos.

a. Es importante el tener conciencia que las decisiones acerca de lo que puede estar o no relacionado o tener o no importancia para un caso, esta basado en valores personales. Se debe permanecer tan objetivo como sea posible en el momento de recoger la información básica sobre la cual se tomará una decisión.

b. Consulte con quien sea necesario para asegurarse de que ha obtenido toda la información necesaria acerca del diagnóstico y pronóstico del caso.

\section{Identifique las principales opciones.}

a. Haga un esfuerzo por identificar todas las principales opciones inclusive aquellas que parezcan moralmente irracionales.

b. Consulte y pida ayuda para garantizar que todas las principales opciones han sido consideradas.

4. Evalúe-analice las opciones de acuerdo con los valores y los principios que están en juego.

a. Comience por informarse acerca de los valores de las partes interesadas procurando tener una idea de las perspectivas y de los valores que cada quien trae a la discusión. Los valores de quienes toman la decisión constituyen lo más importante en un proceso de decisión.

b. Decida si alguna de las opciones viola principios éticos en que todos están de acuerdo son importantes. Elimine aquellas opciones que después del análisis se encuentran como inaceptables para todos.

c. Reexamine las opciones que quedan de acuerdo con los intereses y los valores de cada parte interesada. Algunas alternativas pueden ser combinadas exitosamente.

d. Mida las consecuencias éticas de cada opción, de la opción misma y de los resultados previsibles.

5. Identifique los conflictos éticos y haga lo posible por darles un orden de prioridades.

a. Trate de definir el problema en términos de los principios éticos involucrados, vgr. el deseo de tratar el carcinoma de una paciente (beneficencio) frente a la necesidad de respetar su deseo a no pasar el resto de su vida 
con dolores y en un hospital (autonomía-el no hacer el mal).

b. Analice los principios en que se fundamenta cada uno de los argumentos expuestos. ¿Cuando se examina el conflicto parece que uno de los principios resalta como más importante que los otros? Parecería por lo tanto que uno de los caminos a seguir es mejor que los otros?

c. Tenga en cuenta opiniones respetables acerca de casos similares y decida hasta que punto ellas pueden ser útiles al considerar el problema actual. Busque las diferencias y similitudes morales pertinentes entre este y otros casos. Usualmente se encontrará que el dilema que se enfrenta no es enteramente nuevo y que las consideraciones de otros al resolver dilemas similares pueden ser útiles.

6. Seleccione las opciones que pueden ser justificadas.

Haga un esfuerzo por llegar a una solución racional del problema, una que pueda ser justificada en términos de los principios éticos universalmente aceptados.
7. Reevalúe la decisión una vez que ha actuado. Repita la evaluación de las principales opciones a la luz de la información obtenida durante la implementación de la decisión. ¿Cuál fue la mejor decisión posible? ¿Qué podemos aprender de la discusión y la solución de este problema?

\section{RESUMEN}

A medida que los médicos se familiaricen más con la disciplina de la ética médica, con sus principios fundamentales y sus conceptos y normas, estarán menos emproblemados cuando enfrenten decisiones clínicas que requieran no sólo una gran pericia médica sino también perspicacia en la forma como los valores y metas personales afectan las decisiones. $\mathrm{Al}$ aproximarse a situaciones éticamente complejas en forma lógica y organizada, el médico aumenta las probabilidades de tener en cuenta todos los factores involucrados o pertinentes. El examen minucioso de principios encontrados y de obligaciones en conflicto generalmente inclinarán la balanza y permitirán una solución. Sin embargo, en los dilemas éticos, pocas veces se llega a soluciones claras como el cristal. Lo que debe buscarse al examinar un caso específico, es un punto en el cual, a la luz de los valores y de las metas de las personas involucradas, pueda llegarse a un acuerdo del peso relativo de los principios encontrados y llegar a una decisión.

\section{BIBLIOGRAFIA}

1. President's Commission for the Study of Ethical Problems in Medicine and Biomedical and Behavioral Research. Deciding to forego life-sustaining treatment: a report on the ethical, medical, and legal issues in treatment decisions. Washington, DC: U.S. Government Printing Office, 1983.

2. PELLEGRINO, E.D. The anatomy of clinical-ethical judgements in perinatology and neonatology: a substantive and procedural framework. Semin Perinatol 11(3): 202-209, 1987.

3. BEAUCHAMP, T.L.; CHILDRESS, J.F. Principles of biomedical ethics, 3rd ed. New York: Oxford University Press, 1989.

4. JONSEN, A.R.; SIEGLER, M.; WINSLADE, W.J. Clinical ethics: a practical approach to ethical decisions in clinical medicine. 2nd ed. New York: Macmillan, 1986.
5. Schloendorff v Society of New York Hospitals, 211 NY 125, 126: 105 NE 92-93, 1914.

6. American College of Obstetricians and Gynecologists. Patient choice: maternal-fetal conflict. ACOG Committee Opinion 55. Washington, DC: ACOG, 1987.

7. American College of Obstetricians and Gynecologists. Endorsement of institutional ethics committees. ACOG Committee Opinión 46. Washington, DC: ACOG, 1985.

8. KANOTI, G.A. Ethics and medical-ethical decisions. Crit Care Clin 2(1): 3-12, 1986.

9. ABRAMS, F.R. Bioethical considerations for high-risk pregnancy. In: Abrams RS, Wexler P, eds. Medical care of the pregnant patient, Boston: Little, Brown, 1-12, 1983. 